\title{
Transiform transmitted virus - is there a risk of its acquisition for dental healthcare workers?
}

\author{
Genoprevalence of TT virus among clinical and auxiliary UK dental healthcare workers: a pilot study by C. Bez, \\ G. Lodi, C. Scully and S. R. Porter Br Dent J 2000; 189:554-555
}

\section{Background}

In 1997 a new human hepatotropic virus, TT virus (TTV), was identified. The clinical significance of TT virus infection remains unclear.

\section{Objective}

To undertake an initial evaluation of the genoprevalence of TTV DNA among healthcare workers in the United Kingdom.

\section{Setting \\ A UK dental hospital.}

\section{Study design}

The sera of 53 dental healthcare workers ( 9 dentists, 14 dental nurses and 30 office staff) were tested for the presence of TTV DNA using Polymerase Chain Reaction (PCR).

\section{Results}

TT virus DNA was detected in 3 out of 53 (5.7\%) samples. This frequency of TTV carriage is similar to that of blood donors and healthy persons in the UK.

\section{Conclusion}

The present data suggest that dental healthcare workers in the UK are not at increased risk of nosocomial acquisition of TT virus.
In Brief

- Transiform transmitted virus (TT virus; TTV) is a recently identified hepatotropic virus.

- The present study has examined the possible frequency of carriage of TT virus in a cohort of UK dental healthcare staff to establish if nosocomial transmission of TT virus in a dental setting is likely.

- The frequency of carriage of TT virus in UK dental staff was similar to that of UK blood donors, hence dental healthcare staff in the UK do not have an increased risk of occupationally acquiring TT virus.

\section{Comment}

The development of screening assays for 1 hepatitis $\mathrm{C}$ virus (HCV) was a landmark step in the prevention of post-transfusion non-A, non-B (NANB) hepatitis. Since then, there has been a global effort to search for other infectious agents responsible for post-transfusion NANB hepatitis not caused by HCV. This led to the recent finding of hepatitis G virus ( syn. HGV/GB $\mathrm{V}-\mathrm{C}$ ), which however is not a major agent of post-transfusion hepatitis. In 1997, Japanese workers identified a novel virus, which they called TT virus in patients with post transfusion hepatitis of unknown etiology. Subsequent studies have shown that TTV is widespread and is found in geographically separated human populations (eg from $16 \%$ in Pakistan to $83 \%$ in Gambia), as well as in chimpanzees in Africa (3\%).

Bez and others have now shown for the first time the genoprevalence of TTV in dental healthcare workers in UK. The frequency of TTV carriage in their cohort $(5.7 \%)$ was similar to that of blood donors implying that dental care workers do not run a higher risk of acquiring this virus than their neighbour next door. This reassuring finding, echoing those of medical care workers in other regions would imply that the universal infection control measures, which are currently in place in dentistry, are more than adequate to prevent TTV infection.

There are however, many unanswered questions with regard to TTV transmission. Findings of Bez et al., and others from varied population cohorts in different parts of the world are compatible with the hypothesis that TTV is transmitted mainly in a nonparenteral or community acquired manner, in addition to the blood transfusion route.
As the authors suggest much more work is warranted to identify the transmission routes of TTV and indeed to establish the relationship between the 'viraemia' and the hepatic disease.

Finally, as has been recently pointed out' the acronym TTV is a rather unfortunate coincidence as it has practical implications in calls for routine screening of blood donors for this virus. The letters TT stand for the initials of a patient from whom this virus was isolated in December 1997, in Japan.

\section{Lakshman P Samaranayake}

Chair Professor of Oral Microbiology,

University of Hong Kong

1. Zuckerman AJ. The acronym TTV (letter) Lancet 1999; 353: 952. 